\title{
Racing wheelchair roll stability while turning: A simple model
}

\author{
Rory A. Cooper, $\mathrm{PhD}$ and Michael MacLeish, MS \\ Human Engineering Laboratory, Biomedical Engineering Program, California State University, \\ Sacramento, CA 95819-2694
}

\begin{abstract}
Wheelchair racing is an important modality in the rehabilitation of people with mobility impairments; thus, it plays a significant role in the lives of many wheelchair users. This paper presents simple roll stability analyses for three- and fourwheeled rider/racing wheelchair systems during turning maneuvers. The results suggest that three-wheeled systems may be less stable than four-wheeled systems under certain circumstances. On the other hand, under certain circumstances, four-wheeled systems could be less stable than three-wheeled systems as well. Further study is required to include human factors in determining the optimal relation between stability and controllability.
\end{abstract}

Key words: dynamic stability, human factors, roll stability, statics, wheelchair racing, wheelchair racing controllability.

\section{INTRODUCTION}

Racing wheelchairs have been designed without formal roll stability analysis, thereby leading to some spectacular crashes due to athletes pushing their chairs beyond the stability envelope. Recent crashes have prompted some road-race directors to limit speeds on certain portions of race courses, some to prohibit the use of three-wheeled racing chairs, and many to require the use of helmets. The restrictions on the use of three-wheeled racing chairs has angered several athletes $(1,2)$. With the speeds attainable by wheelchair racers and the persistent pursuit of faster racing wheelchairs, it is a matter of paramount importance that the dynamic stability of racing wheelchairs be examined (3).

Address all correspondence and requests for reprints to: Rory A. Cooper, PhD, Human Engineering Laboratory, Biomedical Engineering Program, California State University, $6000 \mathrm{~J}$ St., Sacramento, CA 95819-2694.
Roll stability analysis is a standard procedure in the design of many vehicles $(4,5)$. The methods used by motor vehicle manufacturers could be incorporated into wheelchair design. Presently, a void exists in published information concerning the dynamic roll stability of racing wheelchairs, and lightweight, standard, and powered wheelchairs. This investigation was concerned with developing simple analysis procedures for evaluating the roll stability of three- and four-wheeled racing chairs during turning maneuvers.

\section{METHODS}

\section{A geometric approach to static stability}

The geometric approach to stability analysis is primarily a qualitative method of examining the static stability of a vehicle. This should assist nonmathematically inclined readers in developing a more intuitive sense of the notion of the stability of complex systems.

When a racing wheelchair is motionless on a flat surface, there are as many points of contact with the surface as there are wheels. If adjacent contact points are connected with lines, the footprint is constructed (Figure 1). The footprint of a three-wheeled chair is a triangle, whereas the footprint of a four-wheeled chair is a rectangle. For a wheelchair to be statically stable (i.e., when the chair and rider are not moving), the center of gravity (c.g.) of the rider/wheelchair system must remain within the footprint. If the system is tilted on a platform, the perpendicular projections of the points of contact (of the wheels with the platform onto the floor) form the new footprint (Figure 2). One might imagine a rider/wheelchair system sitting 


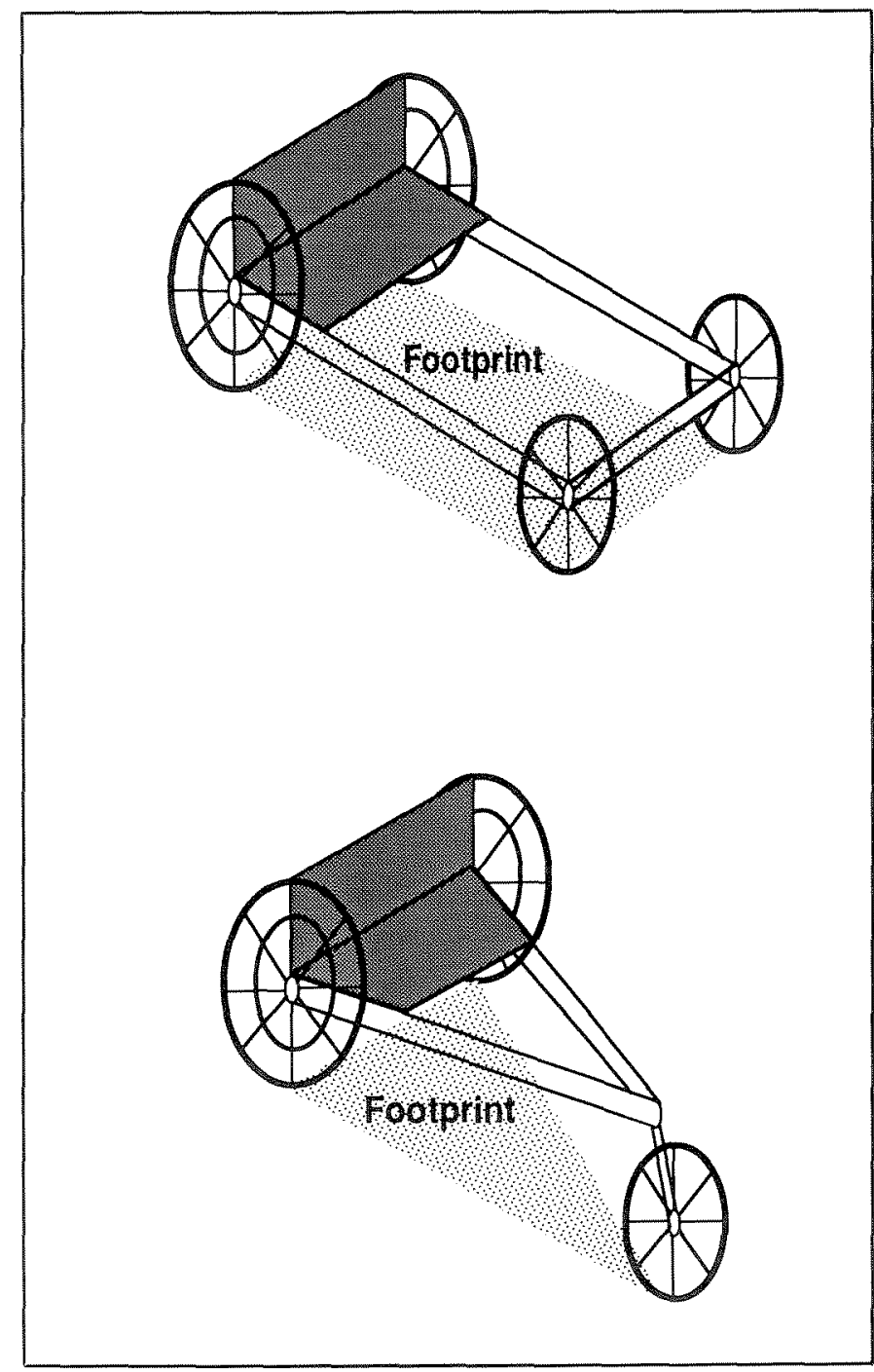

Figure 1.

The concept of a footprint for stability of a vehicle.

on a glass platform that is tilted with respect to the floor: a carpenter's square is placed on the floor directly below the point of contact of each wheel with the glass platform and a corresponding point on the floor. The points on the floor will define the new stability footprint. If the platform were tilted 90 degrees with respect to the floor, the resulting footprint would be a line (i.e., similar to the print of a motionless balanced bicycle). As the rider/wheelchair system is tilted, the footprint becomes smaller and the system becomes more apt to fall. The tilted platform is analogous to a hill. Thus, the rider/wheelchair system is more apt to fall when on a hill, because the footprint (i.e., the region in which the c.g. must remain) is reduced.

The degree of static stability is related to the area of the footprint. All other factors being equal (which is sel-

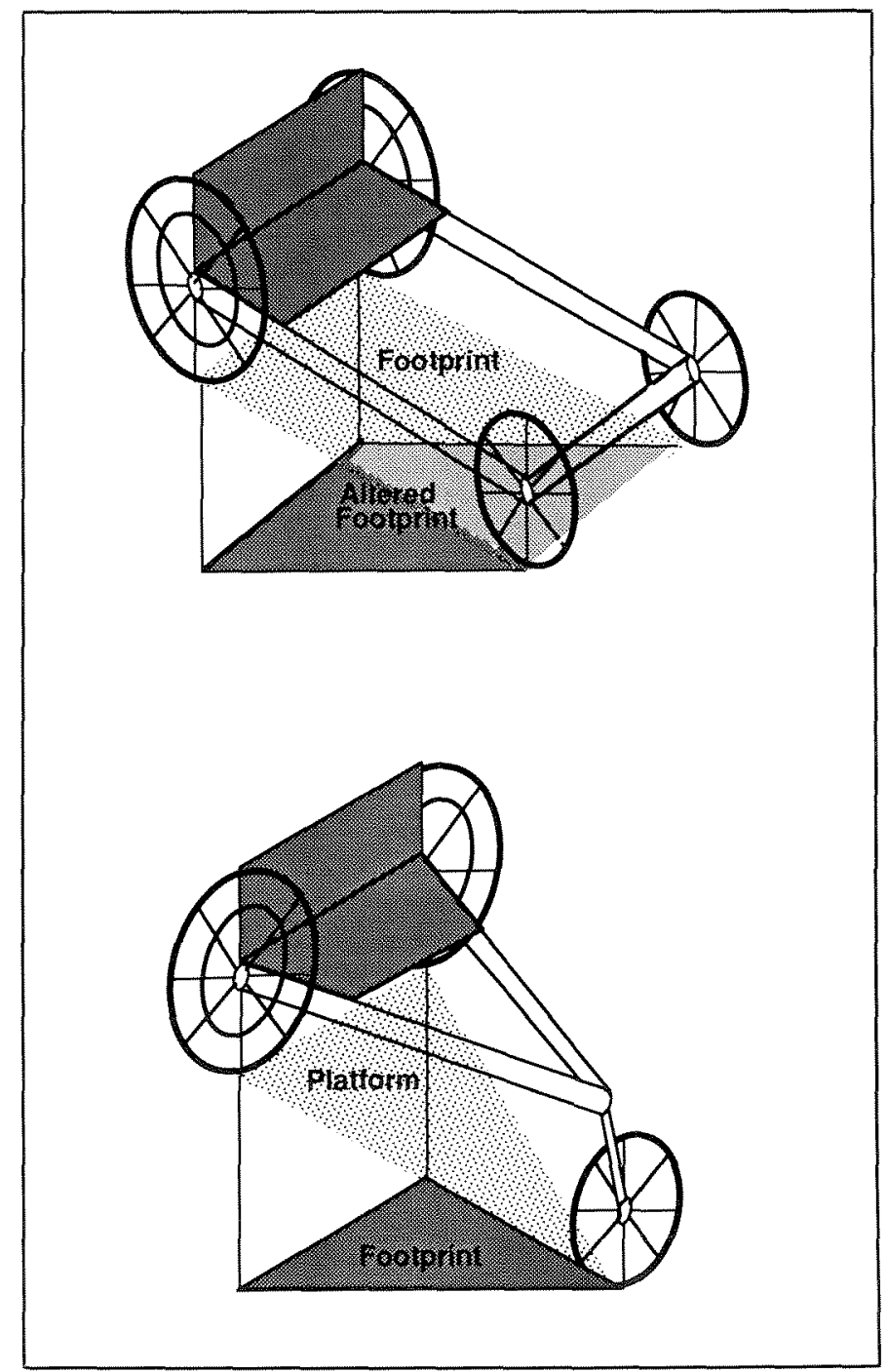

Figure 2 .

Footprint for a three- and four-wheeled racing wheelchair while going downhill, illustrating the reduction of the functional footprint because of the projection onto the horizontal plane.

dom the case), the larger the area of the footprint, the greater the stability. One might interpret this to mean that if the area of the triangular footprint of a three-wheeled chair were to be made the same as the area of the rectangular footprint of a four-wheeled chair, the chairs would be equally stable. This may not be true. If all of the other dimensions were equal, and the c.g. was located in the geometric center of each respective footprint, the chairs would not have the same degree of stability in all directions. The distance of the c.g. from each edge of the respective footprints cannot be equal for three- and fourwheeled chairs because one is a triangle, while the other is a rectangle. 
Up to this point, only the static case has been considered. To examine dynamic stability (i.e., when the system is changing or moving), a more sophisticated approach is required.

\section{An algebraic approach to dynamic stability}

During this investigation, only roll stability while turning was examined while assuming the following:

- The chair does not slip radially

- The road is level and unbanked

- The rider/wheelchair system is rigid.

Figure 3 illustrates the path of the rider/wheelchair system. Figure 4 presents the definitions for the location of the c.g. and the dimensions of the system.

During turning, the radial force acting upon the c.g. at each instant can be described by equation [1].

$$
\mathrm{M}^{\frac{\mathrm{d}^{2} \mathrm{r}}{\mathrm{dt}^{2}}=\mathrm{M}} \frac{\mathrm{v}^{2}}{\mathrm{r}}
$$

Where $\mathrm{M}=$ The mass of the rider/wheelchair system.

$r=$ The radius of the turn.

$\mathrm{v}=$ The speed of the rider/wheelchair system.

$\mathrm{t}=$ Time.

Model with a four-wheeled chair on a level road

It was assumed (as is typically the case) that the front and rear wheels were in line with one another on each side of the chair. Under this assumption, the critical roll stability velocity may be determined by summing the torques acting upon the c.g. of the rider/wheelchair system about a line connecting the outermost front and rear wheels. This is illustrated in equation [2].

$$
\mathrm{MgD}=\mathrm{Mv}_{4}^{2} \mathrm{~L} / \mathrm{r}
$$

The critical velocity for a four-wheeled system can be described by equation [3].

$$
\mathrm{v}_{4}=(\mathrm{gDr} / \mathrm{L})^{1 / 2}
$$

Where $\mathrm{v}_{4}=$ The roll stability critical velocity for a fourwheeled system.

$\mathrm{g}=$ The acceleration due to gravity.

$\mathrm{D}=$ The distance from the point of contact with the road of the outermost wheels to the center of gravity of the rider/wheelchair system with four wheels.

$\mathrm{L}=$ The height above the road of the center of gravity of the rider/wheelchair system.

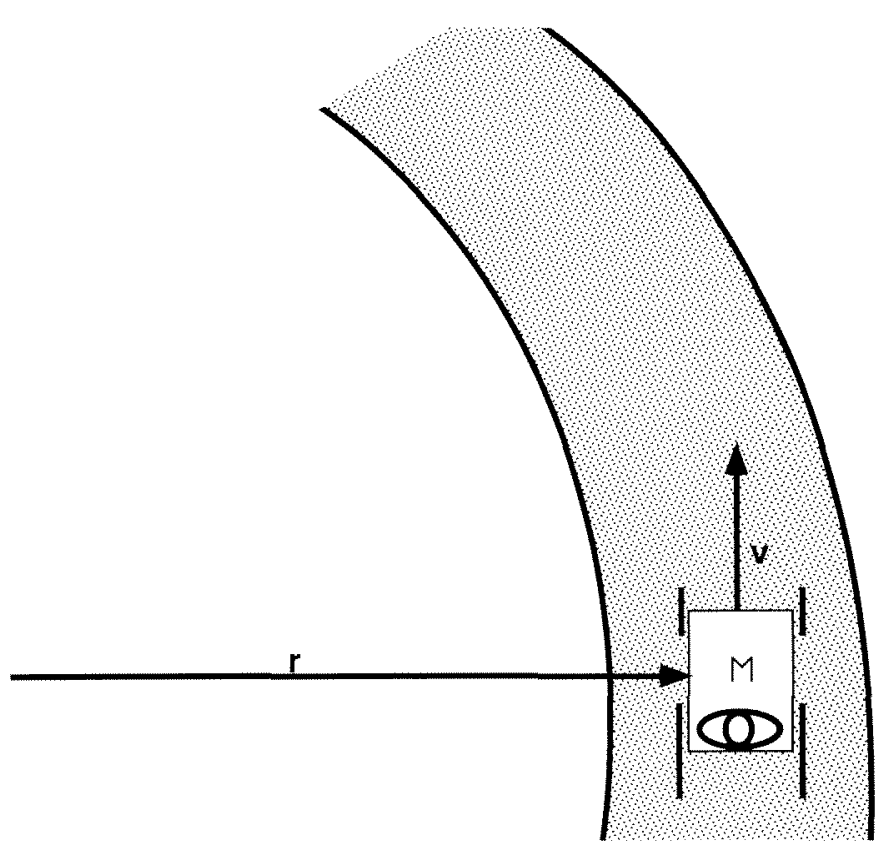

Figure 3.

Depiction of the path of the rider/racing wheelchair system while turning ( $r=$ the radius of the turn, $v=$ velocity of the rider/wheelchair).

The following properties are a result of equation [3]:

$D$ decreases as the chair begins to flip

(the c.g. moves outward);

$\mathrm{L}$ increases until the c.g. is directly over the line connecting the outermost front and rear wheels.

Thus, if speed and curvature are maintained, the system will flip. The greater the difference between the actual speed and the critical speed, the more rapidly the system will flip. Hence, greater skill is required on the part of the rider to control the chair. As the radius of the turn is increased, the critical velocity increases. This can be exploited by the pilot in order to control the racing wheelchair.

\section{Model with a three-wheeled chair on a level road}

Figure 5 presents the definitions of the geometry for a three-wheeled rider/system. The roll stability critical velocity for this system was determined by equating the torques acting upon the c.g. about a line connecting the outermost rear and front wheels (BC). This is illustrated in equation [4]. Therefore, roll stability critical velocity is defined, in this paper, as the velocity at that instant when the torques rotating the racing wheelchair/pilot equal those holding it down. 


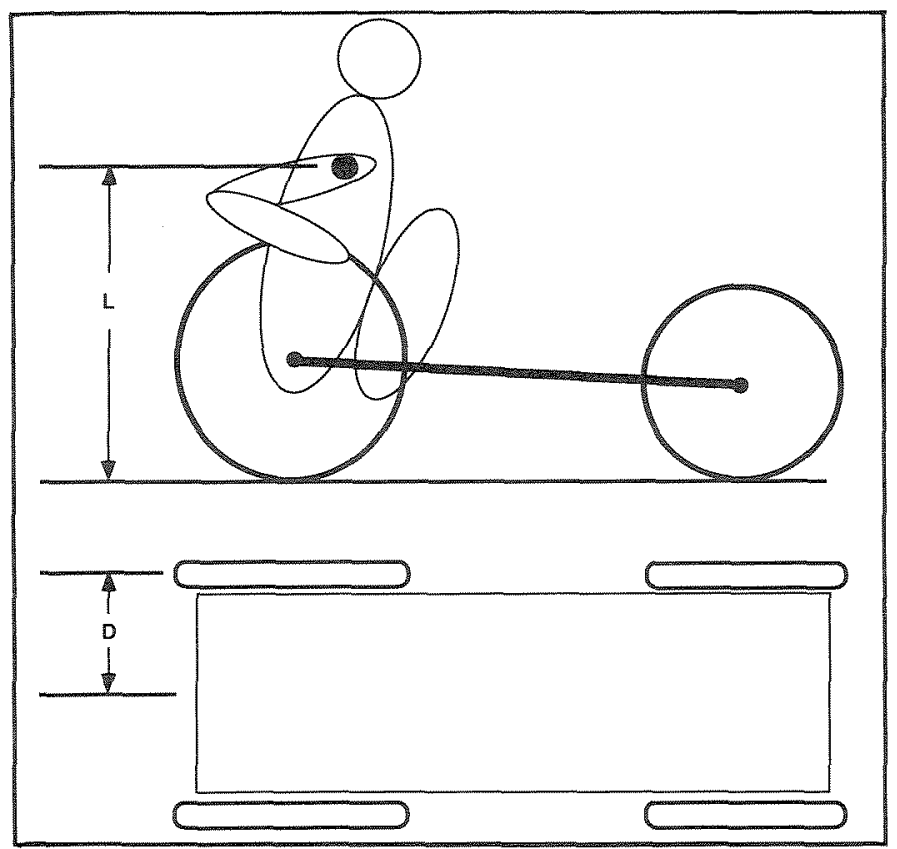

Figure 4.

Pictorial definition of the location of the center of gravity for the rider/racing wheelchair system ( $\mathrm{L}=$ height of the center of gravity above the road, $\mathrm{D}=$ distance from the centerline to the point where a rear wheel makes contact with the road).

$$
\mathrm{MgD}^{\prime}=\mathrm{F}_{\mathrm{p}} \mathrm{L}
$$

Where $\mathrm{D}^{\prime}=$ The horizontal distance of the center of gravity perpendicular to a line connecting the point of contact with the road of the outermost rear and front wheels.

$F_{p}=$ The component of the radial force perpendicular to a line connecting the outermost rear and front wheels $(\mathrm{BC})$.

The perpendicular force $\left(\mathrm{F}_{\mathrm{p}}\right)$ is related to the radial force by equation [5].

$$
F_{p}=F \cos \varphi=\left(M v_{3}^{2} / r\right) \cos \varphi
$$

(Note: $\lim \varphi=0^{0}$ and $F_{p}=F=M v_{3}^{2} / r=M v_{4}^{2} / r$ )

$$
\mathrm{WB}_{3} \hookrightarrow \infty
$$

Where $\mathrm{F}=$ The radial force acting upon the center of gravity of the rider/wheelchair system.

$\varphi=$ The angle between the centerline of the chair and the line connecting the front wheel to a rear wheel.
$v_{3}=$ The critical roll velocity for a three-wheeled system.

For convenience and utility, the distance $\mathrm{D}^{\prime}$ was determined as a function of the frame geometry, and the position of the c.g. of the system, along the centerline of the chair from the line connecting the rear axles. The following equations outline the derivation of these relationships:

$$
\begin{gathered}
\tan \varphi=\mathrm{D} / \mathrm{WB}_{3}=\mathrm{D}^{\prime} / \mathrm{x} \\
\mathrm{x}=\left[\left(\mathrm{WB}_{3}-\mathrm{d}\right)^{2}-\mathrm{D}^{\prime 2}\right]^{1 / 2}=\mathrm{D}^{\prime} \mathrm{WB}_{3} / \mathrm{D} \\
0<\mathrm{d}<\mathrm{WB}_{3} .
\end{gathered}
$$

Where $\mathrm{WB}_{3}=$ Wheelbase of a three-wheeled chair.

$\mathrm{d}=$ Distance of the c.g. along the centerline to the line connecting the rear axles.

$\mathrm{x}=$ The distance from the point where the front wheel touches the ground along a line connecting the front and a rear wheel (BC) to a line perpendicular to $\mathrm{BC}$ passing through the c.g.

Therefore, the equation for $\mathrm{D}^{\prime}$ is as follows:

$$
\mathrm{D}^{\prime}=\mathrm{D}\left(\mathrm{WB}_{3}-\mathrm{d}\right) /\left(\mathrm{D}^{2}+\mathrm{WB}_{3}^{2}\right)^{1 / 2}
$$

(Note: $\lim \mathrm{D}^{\prime}=\mathrm{D}$ which is intuitive)

$$
\mathrm{WB}_{3} \Rightarrow \infty
$$

Equations [5] and [8] were substituted into equation [4] resulting in equations [9] and [10] which lead to the solution of the critical roll velocity for a threewheeled system.

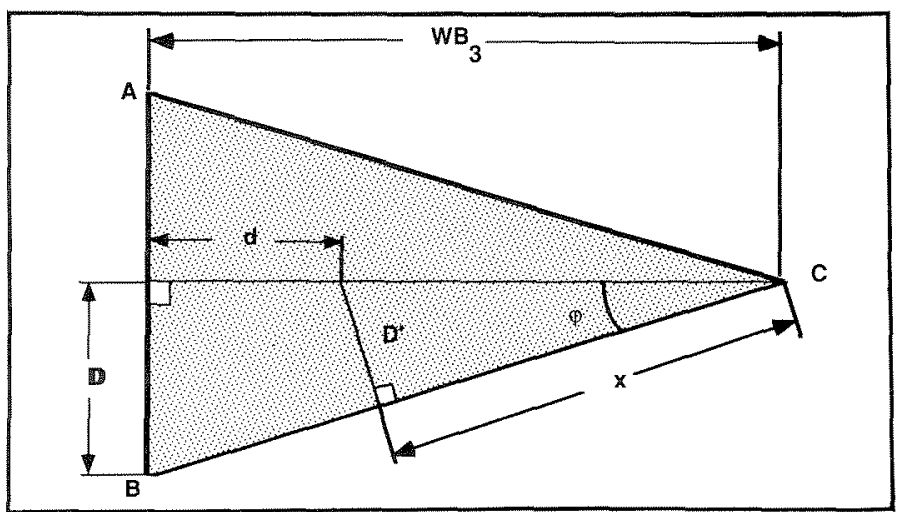

Figure 5.

The frame dimensions used in the dynamic stability analysis for a threewheeled racing chair system. 


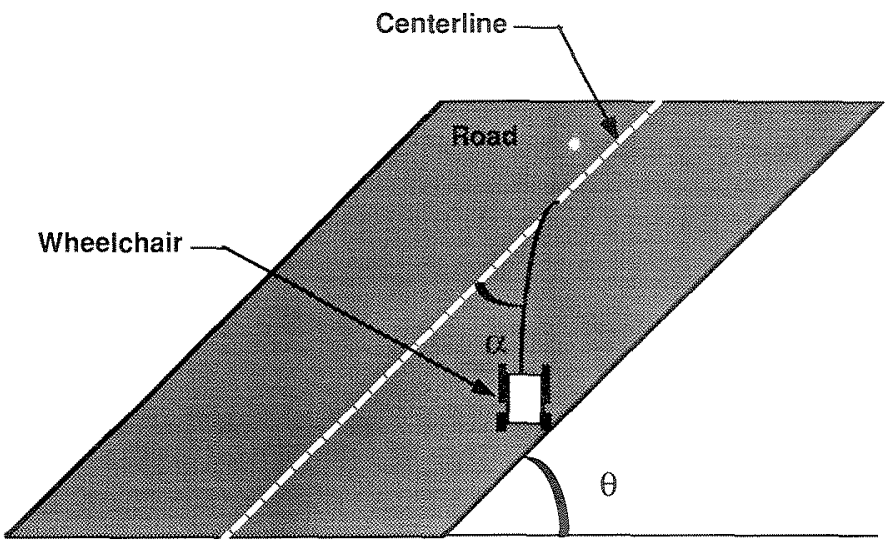

Figure 6a.

A rider/racing wheelchair system turning on a downhill slope.

$$
\operatorname{MgD}\left(\mathrm{WB}_{3}-\mathrm{d}\right) /\left(\mathrm{D}^{2}+\mathrm{WB}_{3}^{2}\right)^{1 / 2}=\left(\mathrm{Mv} v_{3}^{2} \mathrm{~L} / \mathrm{r}\right) \cos \varphi
$$

From the definitions in Figure 5, the relation for the frame angle can be derived.

$$
\cos \varphi=\mathrm{WB}_{3} /\left(\mathrm{D}^{2}+\mathrm{WB}_{3}^{2}\right)^{1 / 2}
$$

Equation [11] describes the roll stability of a simple threewheeled system.

$$
v_{3}^{2}=(\operatorname{grD} / \mathrm{L})\left(\mathrm{WB}_{3}-\mathrm{d}\right) / \mathrm{WB}_{3}
$$

(Note: $\lim v_{3}^{2}=0$, unicycle effect)

$$
\mathrm{d} \subset \mathrm{WB}_{3}
$$

Equation [11] illustrates that as the position of the c.g. moves away from the rear axles, a three-wheeled racing wheelchair becomes less stable. The roll stability of both three- and four-wheeled systems on a flat road has been derived. Next, the roll stability of three- and four-wheeled systems will be extended to going down hills.

Dynamic roll stability while turning on a downhill slope

Analysis of roll stability while turning on a downhill slope requires additional definitions (Figure 6a, and Figure $\mathbf{6 b})$. In this analysis, the assumptions are relaxed by removing the requirement that the road be level. While turning on a downhill slope, the angle of inclination of the hill must be accounted for as well as the radial force due to turning.

\section{The four-wheeled system on a downhill slope}

As before, the roll stability critical velocity may be determined by summing the torques acting upon the c.g. of the rider/wheelchair system about a line connecting the

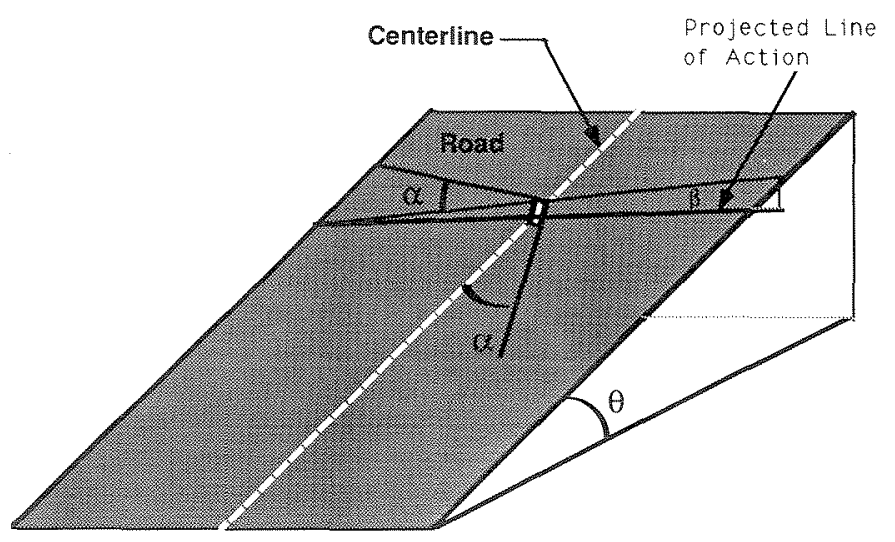

Figure 6b.

Definition of variables for a rider/racing wheelchair system turning on a downhill slope.

outermost front and rear wheels. The sum of the torques about a line between the outermost front and rear wheels is given by equation [12].

$$
\begin{gathered}
M v_{4}^{2} \mathrm{~L} / \mathrm{r}=\mathrm{Mg}\left[\mathrm{D}_{\mathrm{p}}-\mathrm{L}_{\mathrm{p}}\right] \\
\beta=\cos ^{-1} \sqrt{\cos ^{2} \alpha+\left(\sin ^{2} \alpha\right)\left(\cos ^{2} \theta\right)} \\
\mathrm{L}_{\mathrm{p}}=\mathrm{L} \sin \beta, \mathrm{D}_{\mathrm{p}}=\mathrm{D} \cos \beta \\
0 \leq \alpha \leq 90^{\circ}, 0 \leq \theta \leq 90^{\circ}
\end{gathered}
$$

If $\theta=0$, then equation [12] reduces to equation [2]. If

$$
\theta=\frac{\pi}{2}, a=\frac{\pi}{2} \text { then } M v_{4}^{2} L / r+M g L=0
$$

which implies $v_{4}^{2}<0$; thus, the system is unstable, as predicted from static analysis. For equations [12] and [13] the following definitions apply:

$$
\begin{aligned}
& \theta=\text { The angle of inclination of the hill. } \\
& \alpha=\text { Angle of the turn with respect to the center- } \\
& \text { line of the roadway. }
\end{aligned}
$$

Rearranging equation [12] yields the critical roll stability velocity for a four-wheeled system on a downhill slope, equation [14].

$$
v_{4}=\sqrt{\frac{r g}{L}\left(D_{p}-L_{p}\right)}
$$

The three-wheeled system on a downhill slope

With the same definitions as in the previous section, the equation for critical roll stability velocity for a three-wheeled system on a downhill slope is given by equation [15]. 
Journal of Rehabilitation Research and Development Vol. 29 No. 2 Spring 1992

$$
\begin{gathered}
\frac{\mathrm{Mv}_{3}^{2} \mathrm{LWB}_{3} / \sqrt{\mathrm{D}^{2}+\mathrm{WB}_{3}^{2}}}{\mathrm{r}}=\mathrm{Mg}\left(\mathrm{D}_{\mathrm{p} 3}-\mathrm{L}_{\mathrm{p} 3}\right) \\
\mathrm{L}_{\mathrm{p} 3}=\left(\mathrm{LWB}_{3} \sin \beta\right) / \sqrt{\mathrm{D}^{2}+\mathrm{WB}_{3}^{2}} \\
\mathrm{D}_{\mathrm{p} 3}=\left(\mathrm{D}\left(\mathrm{WB}_{3}-\mathrm{d}\right) \operatorname{co} \beta\right) / \sqrt{\mathrm{D}^{2}+\mathrm{WB}_{3}^{2}}
\end{gathered}
$$

As in the case of the four-wheeled system, equation [15] reduces to the level road case when $\theta=0$, and the system is unstable when $\theta=\frac{\pi}{2}, \alpha=\frac{\pi}{2}$. The critical roll stability velocity equation for a three-wheeled system on a downhill slope is presented in equation [16].

$$
v_{3}=\sqrt{\frac{\operatorname{rg}\left(D_{p 3}-L_{p 3}\right) \sqrt{D^{2}+W B_{3}^{2}}}{L W B_{3}}}
$$

\section{RESULTS}

The equations that describe the roll stability for three- and four-wheeled systems can be used to determine the relative stability of three- and four-wheeled racing wheelchair systems. The critical roll stability velocity on a flat roadway for four- and three-wheeled systems were described by equations [3] and [11], respectively. The critical roll stability velocity on a downhill slope for fourand three-wheeled systems were described by equations [14] and [16], respectively; from these equations, the relative velocity of three- and four-wheeled systems can be determined.

\section{Relative stability of three- and four-wheeled rider/ wheelchair systems}

The relative stability of three- and four-wheeled rider/racing wheelchair systems follows directly from equations [3] and [11]

$$
\begin{gathered}
\mathrm{v}_{4}^{2}=(\mathrm{rgD} / \mathrm{L}) \\
\mathrm{v}_{3}^{2}=(\mathrm{rgD} / \mathrm{L})\left(\mathrm{WB}_{3}-\mathrm{d}\right) / \mathrm{WB}_{3}
\end{gathered}
$$

The critical roll stability velocities of three- and fourwheeled systems are related by equation [17]

$$
\frac{v_{3}^{2}}{v_{4}^{2}}=\frac{\left(W_{3}-d\right)}{W_{3}}
$$

which implies that these systems have the following properties:

$$
\begin{aligned}
& \lim _{\mathrm{v}_{3}^{2}=\mathrm{v}_{4}^{2}} \\
& \mathrm{WB}_{3} \Rightarrow \infty \\
& \lim _{\mathrm{d} \Leftrightarrow 0} \mathrm{v}_{3}^{2}=\mathrm{v}_{4}^{2} \\
& \quad \text { (center of gravity chair) }
\end{aligned}
$$

The relative stability of three- and four-wheeled systems was determined by manipulating equations [14] and [16]. Equation [20] presents the relative stability of threeand four-wheeled systems while going downhill.

$$
\frac{v_{3}^{2}}{v_{4}^{2}}=\frac{\left(D_{p 3}-L_{p 3}\right) \sqrt{D^{2}+W B_{3}^{2}}}{W B_{3}\left(D_{p}-L_{p}\right)}
$$

As in the level ground case, equivalence is achieved for certain limiting conditions.

$$
\begin{aligned}
& \lim _{\mathrm{WB}_{3} \leftrightharpoons \infty} v_{3}^{2}=v_{4}^{2} \\
& \lim _{d \hookrightarrow 0} v_{3}^{2}=v_{4}^{2}
\end{aligned}
$$

For many three-wheeled systems, it can be shown that if $\mathrm{d} \leq \frac{1}{3} \mathrm{WB}_{3}$, the critical velocity will be within 10 percent of that for a four-wheeled system. As $\mathbf{d}$ approaches zero, the yaw stability is reduced, making it difficult to control the direction of the chair. For example, using the data collected from 12 experienced wheelchair racers, Table 1 (for $\mathrm{D}$ and $\mathrm{WB}_{3}$ ), and estimated values for the other terms, if $r=20$ meters, $g=9.81$ meters per second (mps) squared, $\mathrm{D}=0.28$ meters, $\mathrm{d}=0.09$ meters, $\mathrm{L}=0.50$ meters, and $\mathrm{WB}_{3}=0.70$ meters, then the estimated critical roll stability velocity for a four-wheeled system is $v_{4}=10.48$ mps, and the estimated critical roll stability velocity for a three-wheeled system is $v_{3}=9.79 \mathrm{mps}$ which represents a 6.6 percent difference. If the same systems were placed on a downhill slope with angle $\theta=2$ degrees, and a turning angle $\alpha=10$ degrees (angle with respect to the fall line), then $\mathrm{v}_{4}=10.43 \mathrm{mps}$ and $\mathrm{v}_{3}=6.81 \mathrm{mps}$, which represents a 34.7 percent difference. As some of these values are only hypothetical in nature, they need to be measured to actually determine roll stability. 
Table 1.

Racing wheelchair and pilot dimensional and anthropometric data $(\mathrm{N}=12)$.

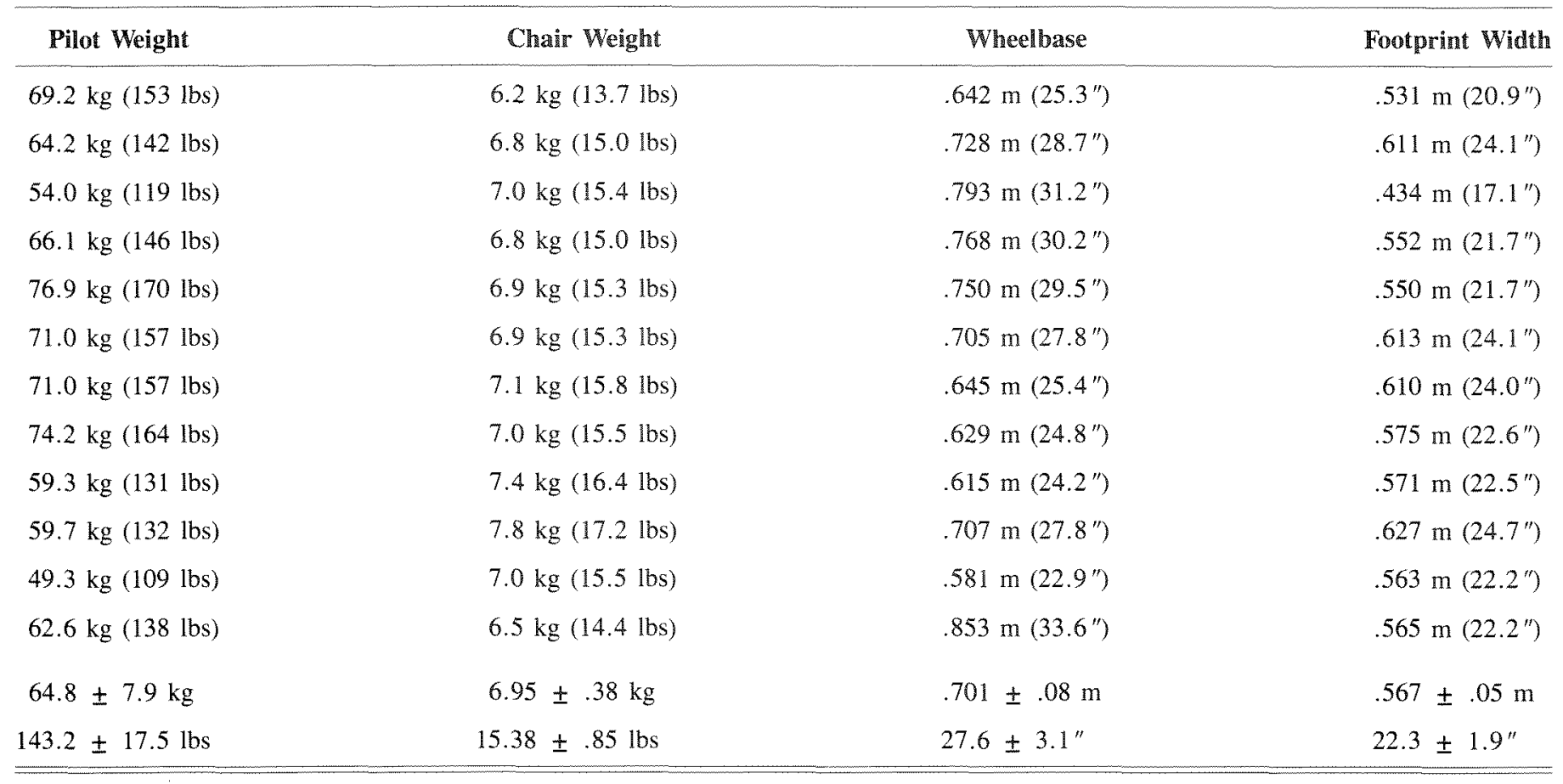

\section{SUMMARY AND CONCLUSIONS}

Static and fixed dynamic stability analyses for racing wheelchairs have been investigated. Only the simple (but important) case of roll stability while turning was investigated. This analysis did not consider an active human controller, nor a multitude of other possible circumstances. Since this was only a preliminary analysis, many other scenarios remain to be investigated, such as road irregularities, wind gusts, flexible system models, and the interaction of other competitors. Further analysis may show that four-wheeled racing chairs have some disadvantages in turns, particularly on a rough surface where they may be less controllable. Often a four-wheeled racing wheelchair rides on only three of its wheels. When this occurs, the stability footprint is an asymmetrical triangular base as opposed to a constant symmetrical triangular base which always exists on a three-wheeled racing wheelchair traveling below the critical velocity. These analyses do, however, provide a foundation for future analysis of roll stability of racing wheelchairs, and a means for estimating the critical roll velocity while turning when given the geometry of the chair and the position of the center of gravity of the rider/racing wheelchair system.

Based upon the assumptions made for these analyses, preliminary results show that, while turning, racing wheelchairs with three wheels may be less stable than those with four. The results show that both three- and four-wheeled chairs are more apt to roll over while turning on a downhill slope than on a level surface. The conditions necessary in order for the dimensions of three- and four-wheeled chairs to yield equal fixed dynamic roll stability properties while turning are presented. This analysis should not be interpreted to mean that three-wheeled racing wheelchairs are unsafe. In the past, some three-wheeled chairs may have been unsafe due to errors in the design of the steering geometry. But, with the proper geometry and human control factors built into three-wheeled racing wheelchairs, they are now safer-with greater dynamic stability while turning. As the pilot's mass is significant with respect to the total mass of the system, the ability to change the c.g. with rapid movement of the upper body is important to the control of a racing wheelchair, just as it is important to the control of a two-wheeled bicycle. The "lean" of the pilot may increase D beyond the half-width of the racing wheelchair. This will have a very significant influence on the control of the chair.

Early in the development of aircraft there was much discussion about the issue of stability (6). Some aircraft designers believed that an aircraft should be very stable 
(i.e., return to its original course and attitude after a disturbance with little effort on the part of the pilot). However, many pilots preferred aircraft which were only marginally stable but gave them greater control over the aircraft. Over time, designers and engineers came to develop a greater understanding of the dynamics of aircraft and now have a better understanding of the human factors involved. As with aircraft, controllability and stability of wheelchairs are competing factors and require engineers: pilots need to make design trade-offs to achieve optimal designs. Further study of this system is required before any real conclusions can be made. Wheelchair/pilot systems are extremely complex; stability and controllability must be determined for changing dynamic situations.

\section{REFERENCES}

1. Clark P. Three wheelers [letter]. Sports 'N Spokes 1988;14(1):6.

2. Dowling J. Three wheelers [letter]. Sports 'N Spokes 1988;14(1):6.

3. Huber JH, LaDuke A. Marathon Mess. Sports 'N Spokes 1987;13(3):36-8

4. Maciejowski JM. Multivariable feedback design. Menlo Park, CA: Addison-Wesley Publishing Co., Inc., 1989.

5. Rosenburg RC, Karnopp DC. Introduction to physical system dynamics. San Francisco, CA: McGraw-Hill Book Company, 1983.

6. Vincenti WG. How did it become 'obvious' that an airplane should be inherently stable? The rise of an engineering truth from a quagmire of human needs, experience and technological compromise. Invention and Technology, 1988;Spring-Summer:50-6.

\section{APPENDIX}

\section{Sample MATLAB Routine for Estimating Wheelchair Roll Stability}

(The following code is to be entered into a computer:)

function $[\mathrm{v} 3, \mathrm{v} 4, \mathrm{v} 3 \mathrm{i}, \mathrm{v} 4 \mathrm{i}]=\mathrm{stable}(\mathrm{L}, \mathrm{D}, \mathrm{d}, \mathrm{WB} 3, \mathrm{r}$, alpha, theta $)$

$\%[v 3, v 4, v 3 i, v 4 i]=$ stable $(\mathrm{L}, \mathrm{D}, \mathrm{d}, \mathrm{WB} 3, \mathrm{r}$, alpha, theta)

\% This function calculates the roll stability of three and four wheeled

$\%$ wheelchairs using a fixed dynamic analysis. This routine is only designed

$\%$ to give a best guess estimate and does not include a number of factors

$\%$ related to stability in reality.

$\% \quad$ v3 $=$ Critical Roll Stability Velocity for a Three Wheeled Chair.

$\% \quad \mathrm{v} 4=$ Critical Roll Stability Velocity for a Four Wheeled Chair.

$\% \quad v 3 i=$ Critical Roll Stability on an Incline for a Three Wheeled Chair.

$\% \quad v 4 i=$ Critical Roll Stability on an Incline for a Four Wheeled Chair.

$\% \quad \mathrm{~L}=$ Height of the Center of Gravity for the Chair/Pilot.

$\% \quad \mathrm{D}=$ Half the Width of the Footprint.

$\% \quad d=$ Distance of Center of Gravity in Front of Rear Axles.

$\% \quad$ WB3 $=$ Wheelbase of Three Wheeled Chairs.

$\% \quad r=$ Radius of Turn.

$\% \quad$ alpha $=$ Turning Angle.

$\% \quad$ theta $=$ Angle of Inclination.

$\mathrm{g}=9.81 ; \%$ Acceleration due to Gravity.

$\mathrm{v} 3=\operatorname{sqrt}(((\mathrm{g} * \mathrm{r} * \mathrm{D} / \mathrm{L}) *(\mathrm{WB} 3-\mathrm{d})) / \mathrm{WB} 3)$;

$\mathrm{v} 4=\operatorname{sqrt}(\mathrm{g} * \mathrm{D} * \mathrm{r} / \mathrm{L})$

$\%$ Convert angles from degrees to radians.

alpha $=($ alpha $/ 360) * 2 *$ pi;

theta $=($ theta/360 $) * 2 * \mathrm{pi}$;

$\%$

beta $=\operatorname{acos}\left(\operatorname{sqrt}\left((\cos (\right.\right.$ alpha $)) \wedge 2+((\sin (\text { alpha })) \wedge 2)^{*}((\cos ($ theta $\left.\left.)) \wedge 2)\right)\right)$;

$\mathrm{Lp}=\mathrm{L} * \sin ($ beta $)$

$\mathrm{Dp}=\mathrm{D} * \cos ($ beta $)$;

$\mathrm{v} 4 \mathrm{i}=\operatorname{sqrt}\left(\left(\mathrm{r}^{*} \mathrm{~g} / \mathrm{L}\right) *(\mathrm{Dp}-\mathrm{Lp})\right)$;

$\mathrm{Lp} 3=(\mathrm{L} * \mathrm{WB} 3 * \sin ($ beta $)) / \operatorname{sqrt}((\mathrm{D} \wedge 2)+(\mathrm{WB} 3 \wedge 2))$;

Dp3 $=\left((\mathrm{D} *(\mathrm{WB} 3-\mathrm{d}))^{*} \cos (\right.$ beta $\left.)\right) / \operatorname{sqrt}((\mathrm{D} \wedge 2)+(\mathrm{WB} 3 \wedge 2))$;

v3i $=\operatorname{sqrt}\left(\left(r^{*} \mathrm{~g}^{*}(\mathrm{Dp} 3-\mathrm{Lp} 3){ }^{*} \operatorname{sqrt}((\mathrm{D} \wedge 2)+(\mathrm{WB} 3 \wedge 2))\right) / \mathrm{L} * \mathrm{WB} 3\right)$;

MATLAB is a software package produced by the Math Works Inc., Sherborn, MA. 